\title{
Pediatric Voice and Swallowing Disorders Related to Vocal Fold Immobility: the Use of Laryngeal EMG
}

\author{
Carissa J. Wentland, $D O^{1}$ \\ Phillip C. Song, $M D^{2}$ \\ Christopher J. Hartnick, $M D^{3, *}$
}

\author{
Address \\ ${ }^{1}$ Department of Pediatric Otolaryngology, University Hospitals, Cleveland Medical \\ Center, Cleveland, $\mathrm{OH}$, USA \\ ${ }^{2}$ Department of Laryngology; Massachusetts Eye and Ear Infirmary, Boston, MA, USA \\ ${ }^{*}, 3$ Department of Pediatric Otolaryngology; Massachusetts Eye and Ear Infirmary, \\ 243 Charles Street, Boston, MA, 02114, USA \\ Email: Christopher_hartnick@meei.harvard.edu
}

Published online: 8 0ctober 2016

(C) Springer International Publishing AG 2016

This article is part of the Topical Collection on Otolaryngology

Keywords Dysphonia $\cdot$ Dysphagia $\cdot$ Vocal cord paralysis $\cdot$ Immobility $\cdot$ Electromyography $\cdot$ Reinnervation

\section{Opinion statement}

Vocal fold immobility is a common cause of voice and swallowing disorders in children. The etiology of vocal fold immobility can be iatrogenic, idiopathic, neurological, or traumatic. Unilateral vocal fold immobility manifests as a weak or breathy voice and swallowing disorders such as aspiration. In contrast, bilateral vocal fold immobility typically presents with stridor or respiratory distress. Treatment for vocal fold immobility is based on symptoms. Patients with respiratory distress due to bilateral vocal fold immobility often require a tracheotomy, vocal cordotomy, arytenoidectomy, arytenoid abduction, or procedures to open the glottic airway. Surgical intervention for unilateral vocal fold immobility can include injection laryngoplasty, type 1 thyroplasty, or laryngeal reinnervation procedures. Timing for intervention with unilateral vocal fold immobility is difficult to determine for iatrogenic, traumatic, or neurologic injuries. Laryngeal electromyography (EMG) can help differentiate a neurologic etiology from vocal fold immobility from arytenoid dislocation. This diagnostic and prognostic information can help determine timing for definitive procedures such as laryngeal reinnervation.

\section{Introduction}

Pediatric voice and swallow disorders are commonly encountered by otolaryngologists. The true incidence of pediatric dysphonia is unknown and thought to be underestimated [1]. Reports have estimated pediatric 
dysphonia to be present in $6-40 \%$ of children [1-3]. Over 1 year's time, $1.4 \%$ of children present with a voice disorder with laryngitis and allergy being the most common diagnoses [1]. Benign vocal fold lesions are the most common anatomic cause of pediatric dysphonia [4]. Swallowing problems can affect $0.9 \%$ of children in a year. The most common etiology of the dysphagia was found to be neurological [1]. Table 1 lists common etiologies of both voice and swallow disorders in children. Extensive discussion of the diagnosis and management of every etiology of pediatric dysphonia and dysphagia is beyond the scope of this review. The remainder of this discussion will focus on the etiology, diagnosis, and treatment of vocal fold immobility (VFI), emphasizing the utility of laryngeal electromyography (EMG).

Vocal fold immobility is a common cause of both dysphonia and dysphagia in children. It is defined as reduction or absence of vocal fold movement in the setting of nerve impairment, cricoarytenoid joint fixation, or laryngeal scarring. Vocal fold immobility is the second most common cause of stridor in a neonate, after laryngomalacia. It represents $10 \%$ of congenital laryngeal problems [5, 6]. Unilateral vocal fold paralysis (VFP) typically presents with dysphonia (commonly with a weak or breathy voice), feeding difficulty, and aspiration due to incomplete glottis closure during swallowing $[\mathbf{7} \bullet]$. Bilateral VFP presents with respiratory distress, stridor, retractions, and/or cyanosis [8]. Dysphonia is less common in bilateral VFP, as the vocal folds are often in paramedian or median position $[8,9]$.

The etiology of vocal fold immobility can be iatrogenic, idiopathic, neurological, or traumatic [10•]. Iatrogenic causes include cardiothoracic surgery, with PDA ligation being one of the most common causes of VFI [9,

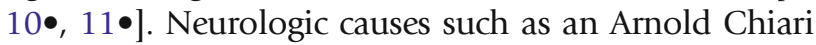
malformation should be considered if there is no obvious history of an inciting event, thus it is important to obtain an MRI on these patients. The MRI should follow the entire course of the recurrent laryngeal nerve to rule out any masses that may cause nerve compression [12].

The workup includes complete history with physical exam and flexible office nasolaryngoscopy. Stroboscopy can be useful but is difficult to perform in young children [3]. Direct laryngoscopy with palpation of the arytenoid complexes to rule out mechanical fixation $[2,3$, $10 \bullet, 12]$. It is important to differentiate neurogenic from mechanical causes of VFI, as this has important prognostic implications and will guide management.

Table 1. List of common causes of voice and swallowing disorders

\section{Voice disorders}

Laryngeal web

Birth trauma

Laryngitis

Vocal fold nodules

Vocal fold cysts

Vocal fold polyps

Postintubation (Subglottic stenosis, vocal fold granuloma, glottic scarring)

Vocal fold avulsion/arytenoid dislocation

Hypothyroidism

Juvenile recurrent respiratory papilloma

Unilateral or bilateral vocal fold paralysis

Velopharyngeal insufficiency

GERD

Neurologic

Functional

Spasmodic dysphonia

Idiopathic

\section{Swallowing disorders}

Laryngeal cleft

Eosinophilic esophagitis

Unilateral vocal fold paralysis

Neurologic

Laryngomalacia 
Laryngeal EMG can be very useful in differentiating neurogenic from mechanical causes of VFI.

Arytenoid dislocation is one of the most common mechanical causes of vocal fold immobility. It is uncommonly reported in the pediatric population. Like adults, the most common etiologies for arytenoid dislocation are intubation and external trauma [13]. Patients present with dysphonia (predominately breathiness), dyspha-

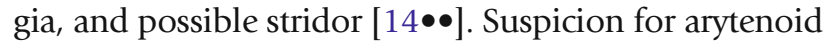
dislocation can be made on flexible fiberoptic nasolaryngoscopy. Reduced vocal fold mobility, vocal fold high mismatch, vocal fold length differences, an absent "jostle" sign and arytenoid edema are all suggestive of arytenoid dislocation [15]. A CT with fine cuts through the larynx can also be helpful [16]. Definitive diagnoses can be made with laryngeal EMG and palpation of the arytenoid under suspension laryngoscopy $[14 \bullet \bullet]$. It is recommended that arytenoid dislocation be treated surgically in a timely fashion to avoid ankyloses of the joint [17]. Spontaneous reduction has been reported and is thought to be accomplished during coughing, vomiting, or other strong movements of the aerodigestive tract $[14 \bullet \bullet]$. Most children require general anesthesia for arytenoid reduction, which uses a moderate amount of force to return the arytenoid to its normal anatomic location [15].

Laryngeal EMG is very useful in determining management strategies for iatrogenic vocal fold immobility. Iatrogenic vocal fold immobility is managed based on the timing of injury and likelihood of recovery. Children can have a greater potential for recovery than adults after iatrogenic VFI. Recovery can be delayed until after a year from the onset of the paralysis. At that time, up to $50 \%$ can recover [10•]. Recovery rates range from 8 to $82 \%$

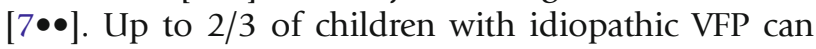
recover (compared to $1 / 3$ in adults) [18]. This recovery can happen as long as 11 years after the onset of the paralysis [10•]. Due to this, the decision about when to perform an intervention can be challenging. In the adult population, it is generally advised to wait a period of at least a year to allow for return of laryngeal function. This timing is less clear in the pediatric population, and some centers recommend delaying permanent interventions for several years $[19,20]$. Laryngeal electromyography (EMG) is a useful tool in predicting the return of laryngeal function. The test can provide useful prognostic indicators and guide further therapy $[20,21,22 \bullet, 23 \bullet$, $24,25 \bullet]$.

\section{Laryngeal electromyography}

Laryngeal EMG was first described by Weddell et al. in 1944 and has been extensively studied by Faaborg-Anderson in 1956 [26-28]. Laryngeal EMG has many uses including nerve monitoring during thyroid and skull base surgery, locating laryngeal muscles during Botox injection for spasmodic dysphonia, and swallow biofeedback [29]. It can also be used to predict the return of vocal fold function after vocal fold paresis $[21,22 \bullet, 23 \bullet, 25 \bullet, 29,30 \bullet \bullet]$. There is still much debate regarding the routine clinical use of laryngeal EMG. In 2009, the Neurolaryngeal Study Group determined that there is little evidence supporting the use of laryngeal EMG, except in determining the location for Botox injection into the laryngeal muscles [30]. A 2016 evidence-based consensus statement from the American Association of Neuromuscular \& Electrodiagnostic (AANEM) Professional Practice Committee recommended the use of laryngeal EMG to clarify treatment decisions for vocal fold immobility thought to be due to disorders of the recurrent laryngeal nerve $[31 \bullet \bullet]$.

\section{Technique}

Laryngeal EMG can be performed either in the office or operating room. The office setting has the benefit of avoidance of anesthesia, and the patient can phonate in an attempt to elicit a response. In-office monitoring can be 
performed with percutaneous monopolar or concentric bipolar needle electrodes or hook wires $[21,24,28]$. The approach is commonly transcervical through the cricothyroid membrane [23•]. In patients where a transcutaneous electrode placement is not an option (due to tracheotomy or difficult landmarks), transoral approaches can be used [22•]. The electrode placement is typically done by an otolaryngologist and the EMG results are often interpreted by a neurologist $[24,29]$. Targeted muscles include the thyroarytenoid, posterior cricoarytenoid, and cricothyroid [21]. Confirmation of correct electrode placement in adults is performed by a motor "gesture"; for the adductors (thyroarytenoid and cricothyroid muscles), the patient vocalizes; for the abductor (posterior cricoarytenoid muscle), the patient inhales briskly through the nose [28]. When unilateral vocal fold immobility is present, the non-affected side can be used as a control [21]. Synkinesis can be identified by having the patient sniff (an abductor function) when monitoring the thyroarytenoid muscle [32]. Evoked laryngeal EMG has been described using a monopolar needle electrode percutaneously to stimulate the external branch of the superior laryngeal nerve and the RLN with recordings of the cricothyroid, thyroarytenoid, posterior cricoarytenoid, and cricothyroid muscles [24].

The optimal timing of laryngeal EMG in adults varies. EMG findings of nerve injury may be masked immediately after injury. Denervation potentials take about 4 to 7 days after injury to be detected and will evolve over several months. Mu and Yang recommended that a new vocal fold paralysis should be evaluated with laryngeal EMG at three intervals: 1, 4-5, and 10-12 weeks after injury [33]. Min et al. suggest that if performed within 6 weeks after injury, the prognostic capability may be increased [21]. The 2016 consensus statement recommends

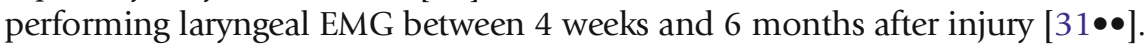

\section{Analysis}

Laryngeal EMG is analyzed for the presence of motor unit potentials, fibrillation potentials, positive sharp waves, and polyphasic potentials. Several reports have suggested that motor unit recruitment is the most valuable predictor of vocal fold movement $[21,23 \bullet]$. Decreased motor unit potentials can be seen as a poor prognostic sign [34]. The 2016 consensus statement has shown that the presence of motor unit potentials increases the likelihood of recovery by $52.6 \%$ $[31 \bullet \bullet]$. Motor unit potentials can appear in just under 4 months in canines after

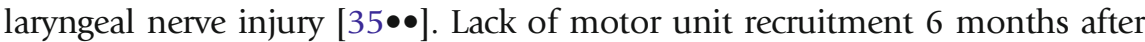
injury offers a poor prognosis [25•]. Partial denervation can present with decreased signals with decreased amplitude and increased latency [24]. However, analysis of the number of motor unit potentials can be subjective and is dependent on accurate needle placement [21].

Fibrillation potentials and positive sharp waves are reliable indicators of laryngeal nerve injuries [24]. However, there is insufficient data about the usefulness of fibrillation potentials and/or positive sharp waves in predicting recovery $[31 \bullet \bullet]$. Denervation can be identified as early as 1 week after injury [24]. In canines, it has been showed that on average, fibrillation potentials disappear in 2 to 3 months but can last up to 4 months [35••]. Synkinesis and aberrant reinnervation can mislead a clinician to expect recovery [36].

The amplification and duration of the EMG waveform can also be useful in providing a quantitative analysis of the EMG [37]. Min et al. [21] found that the 
paralyzed side did have a greater duration of waveform potential; however, it did not find any correlation with the mean duration of the potentials with recovery. Unfortunately, there is limited normative data describing duration and amplification of waveforms.

Regeneration of nerves can be detected as early as 6 weeks after injury [38]. The presence of polyphasic potentials (indicative of neural regeneration) in the first 6 months after injury increases the likelihood of recovery by $44.8 \%$ [31••].

A quantitative approach to interpreting laryngeal EMG uses turns analysis. A turn is defined as a change in signal direction after the peak of a motor unit potential $[34,39]$. As software to perform turns analysis becomes more wide-

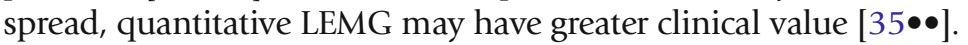

As previously discussed, laryngeal EMG can help differentiate between a non-functioning nerve and cricoarytenoid joint fixation or laryngeal stenosis [25•]. Mechanical fixation will normally present with normal LEMG findings [24].

\section{Predictive accuracy}

Predictive accuracy of laryngeal EMG for determining return of vocal cord function has been reported to be anywhere from 12.8 to $90 \%$ [23•]. Laryngeal EMG is better at predicting persistent paralysis than return of vocal fold function [23•]. The difficulty in predicting recovery arises because it is unknown how much motor unit recovery is necessary to predict return of function [36]. It has been found to shorten the time to intervention because it can accurately predict a poor functional outcome [22•]. Ingle et al. found that laryngeal EMG can shorten observation periods by 2.46 months [25•]. Table 2 shows the results of several studies on the accuracy of laryngeal EMG.

\section{Use in children}

Laryngeal EMG techniques utilized in adults may not be as feasible in children, as most children may have difficulty tolerating percutaneous electrode placement [19]. There are a limited number of pediatric laryngeal EMG studies in the literature [40 $\bullet$. Scott et al. [19] described a simplified approach to laryngeal EMG in children, and we have since modified the procedure.

\section{Procedure}

The patient is initially placed under general anesthesia using propofol and remifentanil hydrochloride. An intraoperative dose of dexamethasone $(0.5 \mathrm{mg} / \mathrm{kg})$ is given. A monopolar grounding electrode is placed subcutaneously into the shoulder. Suspension laryngoscopy is then preformed using the Benjamin Lindholm laryngoscope. Under endoscopic visualization, bipolar double hook wire electrodes are placed into the bilateral thyroarytenoid (TA) and posterior cricoarytenoid (PCA) muscles. Bipolar double hook wire electrodes (Medtronic) record the potential between the two hooks, eliminating the need for a negative wire to be placed externally, which could potentially capture false signals. The non-paretic vocal fold is used as the control. Double hook wire electrodes have the advantage of serving as the patient is then taken out of suspension and allowed to wake up. Vocal fold motion associated with arousal from anesthesia is then captured on the EMG. 
Table 2. Laryngeal EMG study results. This is a list of studies looking at the predictive rate of laryngeal EMG. The findings indicative of laryngeal function and timing of the studies are compared

\begin{tabular}{|c|c|c|c|}
\hline Study & Laryngeal EMG findings & Timing & Predictive rate \\
\hline Hirano et al. [53] & $\begin{array}{l}\text { Presence or absence of action } \\
\text { potentials during voluntary } \\
\text { activity }\end{array}$ & $\begin{array}{l}\text { Less than } 6 \text { months after the } \\
\text { onset of paralysis }\end{array}$ & $\begin{array}{l}69 \% \text { ( } 20 \text { of } 29) \\
7 \text { false positive } \\
2 \text { false negative }\end{array}$ \\
\hline Thumart [54] & Waveform morphology & & $\begin{array}{l}71 \% \text { ( } 81 \text { out of } 114) \\
13 \text { false positive } \\
20 \text { false negative }\end{array}$ \\
\hline $\begin{array}{l}\text { Parnes and } \\
\text { Satya-Murti [34] }\end{array}$ & Waveform morphology & & $\begin{array}{l}88 \% \text { ( } 23 \text { of } 26) \\
3 \text { false positive } \\
\text { No false negative }\end{array}$ \\
\hline Min et al. [21] & $\begin{array}{l}\text { Normal MUP waveform } \\
\text { morphology } \\
\text { Root mean square greater } \\
\text { than } 40 \mathrm{uV} \\
\text { No electrical silence during } \\
\text { voluntary tasks }\end{array}$ & $\begin{array}{l}\text { Less than } 6 \text { months } \\
\text { after onset }\end{array}$ & $\begin{array}{l}89 \%(8 \text { of } 9) \\
1 \text { false negative }\end{array}$ \\
\hline Sittel et al. [22•] & Waveform morphology & $\begin{array}{l}14 \text { days to } 6 \text { months } \\
\text { after onset of paralysis }\end{array}$ & $\begin{array}{l}94.4 \% \text { ( } 68 \text { out of } 72) \text { for } \\
\text { defective recovery } \\
12.8 \%(5 / 39) \text { for complete } \\
\text { recovery }\end{array}$ \\
\hline Munin et al. [23•] & Motor unit recruitment & $\begin{array}{l}21 \text { days to } 6 \text { months } \\
\text { after onset of paralysis }\end{array}$ & $\begin{array}{l}\text { Negative test: } 66.7 \% \\
\text { Positive test: } 80 \%\end{array}$ \\
\hline
\end{tabular}

The nerve integrity monitor (NIM) (Medtronic) is readily available in the operating room. This allows for monitoring of the bilateral TA and PCA at the same time and the electrical parameters for the monitoring is automated and is visually represented and quantified. The other advantage of the NIM system is that it is an integrated unit designed for the operating room. It is shielded from outside electrical interference and has cord lengths long enough to remain out of the surgical field. Conventional diagnostic electromyograms such as the Natus system (Natus Medical Incorporated) have more flexibility in electrical measurements for diagnostic capacity and offer auditory feedback. For instance, in diagnostic EMG, electrical silence can indicate that the electrodes are placed in fat. White noise is heard when the electrode is in air and not contacting tissue. Crackles are heard when the electrode is placed in muscle. The crackles get sharper when the electrode is deeper in muscle. Staccato or "popcorn" sounds are heard when fibrillation potentials are present. Disorganized sounds or "firecrackers" are heard with polyphasic potentials. A recording system is useful to capture audio and video to review after the case is complete. These recordings can then be reviewed by a neurologist who may not be present in the operating room. Figure 1 shows placement of the hook wire electrodes and what is seen on both the NIM and Natus system.

The transoral approaches with children require a knowledgeable and cooperative operating room team. The surgical plan should be discussed before the 


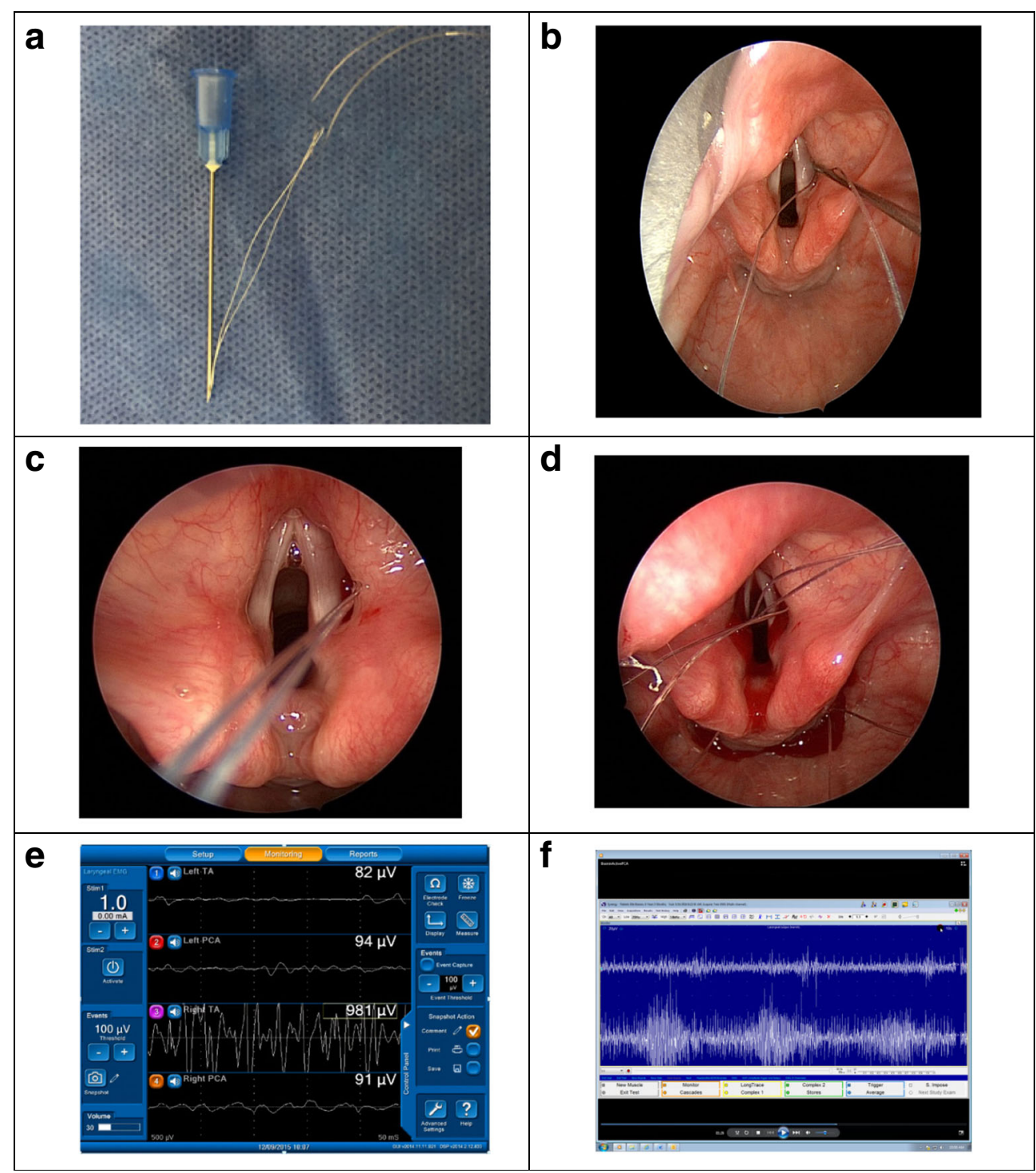

Fig. 1. Transoral laryngeal EMG in children using hook wires. a The hook wire inside the hollow placement needle. $\mathbf{b}$ Placement of the hook wire into the right thyroarytenoid muscle using placement needle. c After the needle is withdrawn, the electrode placement into the right thyroarytenoid muscle. $\mathbf{d}$ Four separate wires into the bilateral thyroarytenoid and posterior cricoarytenoid muscles. e The NIM system (Medtronic) demonstrating a left vocal fold paralysis. $\mathbf{f}$ The Natus system showing a left vocal fold paralysis.

start of the case. It is essential to have a competent pediatric anesthesiologist. It is important to have spontaneous ventilation throughout the case. The patient then needs to be truly awakened with the hook wire electrodes in place to get accurate recordings.

LEMG interpretation is subjective [29]. Improper placement of electrodes can affect the EMG waveforms causing decreased amplitude or duration, 
and even no detection of activity $[21,29]$. It is difficult to discern whether the absence of muscle activity on EMG is secondary to complete vocal cord paralysis or malplacement of the electrode [22•]. We feel that direct visualization of the larynx with a transoral approach is helpful in confirmation that the electrodes are in the proper place.

\section{Management}

Management is determined based on the level of severity of the breathing, voice, or swallowing [40•]. Bilateral VFP can initially be managed with a tracheotomy in the case of respiratory distress. Selective patients who do not exhibit respiratory compromise can be closely monitored [19]. Spontaneous recovery can be observed, with the highest recovery rate being with idiopathic etiology [10•]. Other procedures for bilateral VFP are vocal cordotomy, arytenoidectomy, arytenoid abduction, or arytenoideopexy.

Unilateral vocal fold paralysis presents with voice and swallowing problems. Concern about aspiration and airway protection is high in younger children, and voice concerns escalate as the child matures [41 $\bullet$ ]. Dysphonia can significantly affect a child's quality of life [42]. The low voice volume may lead to much frustration because children cannot be understood in noisy environments or if they are at a distance from others. Also, due to incomplete glottic closure, children require a higher respiratory effort to generate voice which may lead to fatigue [41•]. Conservative management of unilateral VFP includes optimizing speech and swallow techniques to minimalize aspiration. Treatment of gastroesophageal reflux disease (GERD) is recommended [10•].

Voice therapy can be successful in some patients [43]. Up to $40 \%$ of children continue to have symptomatic vocal fold immobility after the recommended observation period, thus surgical treatment options need to be considered $[7 \bullet \bullet, 44 \bullet \bullet]$. Treatment timing should be based on the severity of the handicap caused by the vocal fold immobility $[7 \bullet \bullet]$. Surgical treatment options include injection laryngoplasty, thyroplasty, arytenoid adduction, cricothyroid joint subluxation, or laryngeal reinnervation $[44 \bullet \bullet, 45 \bullet]$.

Injection laryngoplasty involves injecting material into the vocal fold for medialization. Materials used include absorbable gelatin sponge (Gelform; Pfizer Inc.), micronized acellular dermis (Cymetra; LifeCell), sodium carboxymethycellulose gel (Prolaryn Gel, Merz), bovine collagen, calcium hydroxylapatite (Prolaryn Plus, Merz), hydrated gelatin powder, crosslinked stabilized hyaluronic acid (Restylane, Medicis and Juvederm, Allergan), or autologous fat $[44 \bullet \bullet, 46]$. The procedure can be done through a variety of anesthesia methods including total intravenous (IV) anesthesia with spontaneous ventilation, endotracheal intubation,

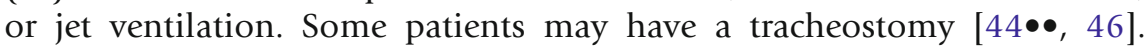
The authors prefer anesthesia with spontaneous ventilation because in a 
child's small airway, an endotracheal tube can occupy a large portion of the airway, making it difficult to assess the extent of medialization. The length of effect varies due to injection material used and patient characteristics. Often, patients will only require one injection due to spontaneous recovery or development of compensatory mechanisms. Complications of injection can include granuloma formation or over injection, leading to airway obstruction $[10 \bullet 44 \bullet \bullet]$.

Type 1 thyroplasty has been described in children using polytetrafluoroethylene implants (GORE-TEX; W. L. Gore \& Associates) or polymeric silicone (Silastic; Dow Corning) [47]. Teenage patients may be mature enough to tolerate the procedure under local anesthesia with

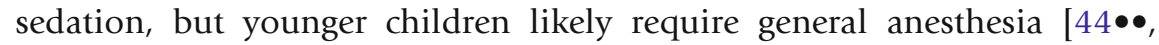
$45 \bullet, 47,48]$. A laryngeal mask airway with fiberoptic endoscopy may be useful for airway management for children requiring type 1 thyroplasty [45•]. It is important to consider the lower position of the true vocal fold in children. This requires a more inferior thyroplasty [47]. Some feel that the immature laryngeal cartilage is too soft to support the implant, and there is a possibility of the implant shifting as the child grows $[41 \bullet]$.

Many children cannot tolerate vocal fold medicalization procedures under sedation with local anesthesia [45•]. Pediatric vocal fold anatomy changes at children grow [49], therefore procedures such as thyroplasty or arytenoid adduction may not be stable throughout the child's growth period [45•]. Also, since pediatric vocal fold motion can spontaneously recover up to 4 years after onset [10•], it may be prudent to avoid more invasive procedures.

Procedures such as arytenoid adduction or cricothyroid joint subluxation should be delayed until after a child's larynx has approached adult size. Even after the larynx has finished growing, these procedures can be more useful in providing fine adjustments to patients who have had other procedures such as thyroplasty or laryngeal reinnervation [45•, $50 \bullet]$.

Reinnervation of the larynx can be accomplished with an ansa cervicalis to recurrent laryngeal nerve anastomosis $[50 \bullet$, 51]. The procedure will medialize the vocal fold and provide baseline tone, but it will not restore the motion of the vocal fold [41•]. The advantage of the procedure is that it does not alter the laryngeal framework, and further procedures can be performed after the child had fully grown [45•]. There is also no need for foreign material that could potentially cause a reaction. The results of the procedure are expected to last a lifetime [41•]. The disadvantage is that it abolishes any chance of spontaneous recovery, since the recurrent laryngeal nerve is sectioned as part of the procedure [45•]. Improvement of symptoms was noted in most patients at 3-7 months $[44 \bullet \bullet, 45 \bullet$. Stable results can be seen a year after surgery [41•]. Therefore, the authors recommend suspension 


\section{Future directions}

laryngoscopy with injection laryngoplasty at the time of surgery to provide glottic competence until reinnervation has occurred. Older children have been shown to have improvement in voice quality, loudness, and pitch range $[41 \bullet, 51]$. The optimal timing for laryngeal reinnervation is not known, but it is important to do before the vocal fold atrophies [41•]. Smith et al. reported voice improvement with the procedure being performed anywhere from 0.8 to 15.2 years after injury. They found no correlation with age at surgery, but there was a slight negative correlation in duration between injury and time of surgery

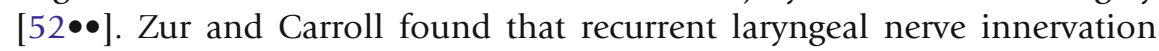
has better acoustic, stroboscopic, and perceptual results than injection or observation [51]. At our center, laryngeal EMG is used before any reinnervation procedure, to assess the tracing for lack of motor unit potentials. We feel it is important to assess the vocal fold to assure there is no muscle atrophy, as reinnervation requires viable muscle.

\section{Conclusions}

A standardized approach to performing and reading laryngeal EMG still needs to be developed. Currently, centers performing laryngeal EMG all use individualized techniques and look for different findings on the EMG. A multicenter trial with the same approach and outcomes would be valuable.

Techniques for refinement of electrode placement need to be developed. One thought is to use ultrasound and image guidance to help improve needle localization $[31 \bullet \bullet]$.

Reports on laryngeal reinnervation have not described using laryngeal

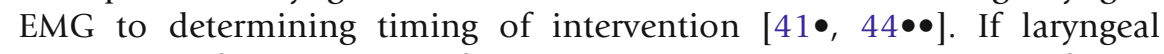
EMG is performed early after injury, and negative prognostic factors (lack of motor unit potentials, fibrillation potentials, positive sharp waves) are seen, laryngeal reinnervation could possibly be performed early to prevent muscle atrophy.

- Laryngeal EMG is useful in determining the timing of intervention for unilateral pediatric vocal fold immobility.

- Conservative options include voice and swallow therapy as well as management of GERD.

- Surgical treatment for unilateral vocal fold immobility includes injection laryngoplasty, type 1 thyroplasty, and laryngeal reinnervation (ansa cervicalis to recurrent laryngeal nerve anastomosis).

- Decision regarding the type of surgical intervention is patient specific. Injection laryngoplasty usually only has temporary effects. Type 1 thyroplasty is more useful in older children whose larynx has fully grown. Laryngeal reinnervation is effective and can be a more permanent solution. 
- Results for laryngeal reinnervation may not be seen for up to 6 months following the procedure, therefore the authors recommend concurrent injection laryngoplasty.

\section{Compliance with Ethical Standards}

\section{Conflict of Interest}

The authors have nothing to disclose. There are no conflicts of interest. There was no financial source for this document.

\section{References and Recommended Reading}

Papers of particular interest, published recently, have been highlighted as:

- Of importance

-• Of major importance

1. Bhattacharyya N. The prevalence of pediatric voice and

10. Daya H, Hosni A, et al. Pediatric vocal fold paralysis: a swallowing problems in the United States. Laryngoscope. 2015;125:746-50.

2. Possamai V, Hartley B. Voice disorders in children. Pediatr Clin N Am. 2013;60:879-92.

3. Smith M. Care of the child's voice: a pediatric otolaryngologist's perspective. Semin Speech Lang. 2013;34(2):63-70.

4. Kilic MA, Okur E, et al. The prevalence of vocal fold nodules in school age children. Int J Pediatric Otolaryngol. 2004;68:409-12.

5. Grundfast K, Harley E. Vocal cord paralysis. Otolaryngol Clin N Am. 1989;22:569-97.

6. Holinger PH, Brown WT. Congenital webs, cysts, laryngoceles, and other anomalies of the larynx. Ann Otol Rhinol Laryngol. 1967;76:744-52.

7.• Jabbour J, Martin T, et al. Pediatric vocal fold immobility: natural history and the need for long-term follow-up. JAMA Otlaryngol Head Neck Surg. 2014;140(5):428-33.

This article looks at at the history and course of vocal fold immobility in children. It gives a great overview of the common etiologies and symptoms, followed by ways to manage symptoms. The authors feel this gives the reader a great understanding of the etiology, progression, and management of vocal fold immobility.

8. Funk RT, Jabbour J, Robey T. Factors associated with tracheotomy and decannulation in pediatric bilateral vocal fold immobility. Int J Pediatr Otolaryngol. 2015;79:895-9.

9. Chen EY, Inglis AF. Bilateral vocal cord paralysis in children. Otolaryngol Clin North Am. 2008;41(5):889-901. long-term retrospective study. Arch Otolaryngol Head Neck Surg. 2000;126(1):21-5.

This article looks at the experience with vocal fold paralysis from the Great Ormond Stree hospital for Children. The authors feel this article does a great job discussing the distribution of etiologies and prognostic outcomes.

11. Truong M, Messner A, et al. Pediatric vocal fold paralysis after cardiac surgery: rate of recovery and sequelae. Otolaryngol Head Neck Surg. 2007;137:780-4.

This article discussed the rate of recovery of pediatric vocal fold paralysis after cardiac surgery. The authors feel this is important because iatrogenic causes are one of the most common etiologies of pediatric vocal fold immobility. It is curitical to understand the rate of recovery and sequelae.

12. Setlur J, Hartnick CJ. Management of unilateral true vocal cord paralysis in children. Curr Opin Otolaryngol Head Neck Surg. 2012;20:497-501.

13. Paulsen FB, Rudert HH, Tillmann BN. New insights into the pathomechanism of postintubation arytenoid subluxation. Anesthesiology. 1999;91:659-66.

14.• Mallon AS, Portnoy JE, et al. Pediatric arytenoid dislocation: diagnosis and treatment. J Voice. 2014;28(1):115-22.

This article looks at the diagnosis and treatment of arytenoid dislocation in children. Etiology, symtpoms, diagnosis, and management are discussed. The authors feel it is important to have arytenoid dislocation as a differential diagnosis for pediatric vocal fold immobility, and it should be ruled out before any surgical management of the other etioloties of vocal fold immobility.

15. Sataloff FT, Bough D, et al. Arytenoid dislocation diagnosis and treatment. Laryngoscope. 1994;104:1353-61. 
16. Hiramatsu H, Tokashiki R, et al. New approach to diagnose arytenoid dislocation and subluxation using three-dimensional computed tomography. Eur Arch Otorhinolaryngol. 2010;267:1893-903.

17. Stack Jr BC, Ridley MB. Arytenoid subluxation from blunt laryngeal trauma. Am J Otolaryngol. 1994;15:68-73.

18. Dambrose EJ, Huang RY, et al. Lack of evoked laryngeal electromyography response in patients after a clinical diagnosis of vocal cord paralysis. Ann Otol Rhinol Laryngol. 2001;110:815-9.

19. Scott A, Chong P, et al. Intraoperative laryngeal electromyography in children with vocal fold immobility: a simplified technique. Int J Pediatr Otolaryngol. 2008;72(1):31-40.

20. Jacobs IN, Finkel RS. Laryngeal EMG in the management of vocal cord mobility problems in children. Laryngoscope. 2002;112:1243-8.

21. Min YB, Finnegan EM, et al. A preliminary study of the prognostic role of electromyography in laryngeal paralysis. Otolaryngol Head Neck Surg. 1994;111(6):770-5.

22. Sittel C, Stennert E, et al. Prognostic value of laryngeal electromyography in vocal fold paralysis. Arch Otolaryngol Head Neck Surg. 2001;127:155-60.

This article looks athe value of laryngeal electromyography in determining the recovery after acute vocal fold paralysis from a variety of causes, with thyroid surgery and idiopathic being the most common causes. The authors feel this article is valuable in illustrating that laryngeal EMG predicted defective recovery correctly in $94.4 \%$ of cases. Prognosis for complete recovery was only accurate in $12.8 \%$ of cases.

23. Munin MC, Rosen CA, Zullo T. Utility of laryngeal electromyography in predicting recovery after vocal fold paralysis. Arch Phys Med Rehabil. 2003;84:1150-3.

This article looks at the value of laryngeal EMG in predicting recovery after vocal fold paralysis. It then describes the specific elements of the EMG that are most predictive of outcome. The authors feel it is important to understand the different components of laryngeal EMG and this is a great reference for the reader to get a further understanding of how each component can predict recovery.

24. Xu W, Han D, et al. Value of laryngeal electromyography in diagnosis of vocal fold immobility. Ann Otol Rhinol Laryngol. 2007;116(8):576-81.

25. Ingle JW, Young VN, et al. Prospective evaluation of the clinical utility of laryngeal electromyography. Laryngoscope. 2014; 124:2745-9.

This great article looks at the prospective use of laryngeal EMG with blinded examiners. The authors feel this is important to illustrate how the diagnosis and treatment plans for vocal fold paralysis can change based on the use of laryngeal EMG.

26. Weddell G, Feinstein B, Pattle RE. The electrical activity of voluntary muscle in man under normal and pathological conditions. Brain. 1944;67:178-257.

27. Faaborg-Anderson K, Buchthal F. Action potentials from internal laryngeal muscles during phonation. Nature. 1956;177:340-1.

28. Hillel AD. The study of laryngeal muscle activity in normal human subjects and in patients with laryngeal dystonia using multiple fine-wire electromyography. Laryngoscope. 2001;111:1-47.

29. Woo P. Laryngeal electromyography is a cost-effective clinically useful tool in the evaluation of vocal fold function. Arch Otolaryngol Head Neck Surg. 1998; 124:472-5.

30. Blitzer A, Crumley RL, et al. Recommendations of the neurolaryngeal study group on laryngeal EMG. Otolaryngol Head Neck Surg. 2009;140:782-93.

31.• Munin MC, Heman-Ackah YD, et al. Consensus statement: Using laryngeal electromyography for the diagnosis and treatment of vocal cord paralysis. Muscle Nerve. 2016;53(6):850-5.

This is an evidence based consensus statement for the use of laryngeal EMG. It discusses the components of laryngeal EMG, including voluntary motor unit potential recruitment, polyphasic motor unit potentials, positive sharp waves, fibrillation potentials, and dynkinesis. The authors feel it is important to understand these components and know what they mean in terms of prognosis. Also, the article emphasizes how laryngeal EMG can alter clinical management decisions.

32. Statham MM, Rosen CA, et al. Electromyographic laryngeal synkinesis alters prognosis in vocal fold paralysis. Laryngoscope. 2010;120:285-90.

33. Mu L, Yang S. An experimental study on the laryngeal electromyography and visual observations in varying types of surgical injuries to the unilateral recurrent laryngeal nerve in the neck. Laryngoscope. 1991;101:699-708.

34. Parnes S, Satya-Murti SM. Predictive value of laryngeal electromyography in patients with vocal cord paralysis of neurogenic origin. Laryngoscope. 1985;95:1323-6.

35.• Paniello RC, Park AM, et al. Recurrent laryngeal nerve recovery patterns assessed by serial electromyography. Laryngoscope. 2016;126:651-6.

This article looks at the change in laryngeal EMG findings over the recovery process. It used canines to serially follow recurrent laryngeal nerve injuries with laryngeal EMG. The authors feel this is a great article that illustrates the imporatance of timing of laryngeal EMG.

36. Smith LJ, Rosen A, et al. Quantitative electromyography improves prediction in vocal fold paralysis. Laryngoscope. 2012;122:854-9.

37. Faaborg-Anderson K. Electromyographic investigation of intrinsic laryngeal muscles in humans. Acta Physiol Scand. 1957; suppl140:1-148.

38. Hirano I, Hirano M, Tomita H. Electromyographic investigation of human vocal cord paralysis. Ann Otol Rhinol Laryngol. 1968;77:296-304.

39. Statham MM, Rosen CA, et al. Quantitative laryngeal electromyography: turns and amplitude analysis. Laryngoscope. 2010;120:2036-41.

40. Maturo SC, Braun N, et al. Intraoperative laryngeal electromyography in children with vocal fold immobility. Arch Otolaryngol Head Neck Surg. 2011;137(12):1251-7.

This article specifically looks at laryngeal EMG in children. It copares the results of larygneal EMG with flexible fiberoptic exam. The authors feels this article is important because it 
shows that laryngeal EMG is safe in chidren, and it can be useful in determining the liklihood of recovery of recurrent laryngeal nerve function.

41. Smith ME, Nelson R, Houtz D. Laryngeal reinnervation for paralytic dysphonia in children younger than 10 years. Arch Otolaryngol Head Neck Surg. 2012;138(12):1161-6.

This article describes laryngeal reinnervation in children younger than 10 years. The authors feel it is important to understand the indication, techniques, and expectations. The article emphasizes that the vocal fold returns to baseline tone but does not have motion.

42. Sajisevi M, Cohen S, Raynor E. Pediatrician approach to dysphonia. Int J Pediatr Otolaryngol. 2014;78(8):1365-7.

43. Schindler A, Bottero A, et al. Etioloty, pathophysiology, treatment choices, and voice results for unilateral adductor vocal fold paralysis: a 3 year retrospective. J Voice. 1999;13(4):618-21.

44.• Butskiy O, Mistry B, Chadha N. Surgical interventions for pediatric unilateral vocal cord paralysis. JAMA Otolaryngol Head Neck Surg. 2015;141(7):654-60. This is a summary of the available evidence for the surgical management of pediatric unilateral vocal fold paralysis. The authors feel it is important to understand the surgical options and relative safety of these interventions.

45. Sipp JA, Kerschner J, et al. Vocal fold medialization in children: injection laryngoplasty, thyroplasty, or nerve reinnervation? Arch Otolaryngol Head Neck Surg. 2007;133(8):767-71.

This article reviews the available options for vocal fold medialization in children. The authors feel it is valuable to know that injection laryngoplasty, thyroplasty, and nerve reinnervation can be performed safely in children with good outcomes. This also describes the advantages and disadvantages of each procedure.

46. Cohen MS, Mehta DK, et al. Injection mediatization laryngoplasty in children. Arch Otolaryngol Head Neck Surg. 2011;137(3):264-8.
47. Thompson Link D, Rutter MJ, et al. Pediatric type I thyroplasty: an evolving procedure. Ann Otol Rhinol Laryngol. 1999;108:1105-10.

48. Gardner GM, Altman JS, Balakrishnan G. Pediatric vocal fold medialization with silastic implant: intraoperative airway management. Int J Pediatr Otorhinolaryngol. 2000;52(1):37-44.

49. Hudgins PA, Siegel J, et al. The normal pediatric larynx. AJNR Am J Neuroradiol. 1997;18(2):239-45.

50. Zur KB. Recurrent laryngeal nerve reinnervation for unilateral vocal fold immobility in children. Laryngoscope. 2012;122:S82-3.

This article discusses recurrent laryngeal nerve reinnervation with use of laryngeal EMG in 10 pediatric patients with dysphonia secondary to vocal fold immobility. The authors feels this article illustrates that recurrent laryngeal nerve reinnervation is a great long term management strategy of vocal fold immobility in children.

51. Zur KB, Carroll LM. Recurrent laryngeal nerve reinnervation in children: acoustic and endoscopic characteristics pre-intervention and post-intervention. A comparison of treatment options. Laryngoscope. 2015;125:S1-15.

52.• Smith ME, Houtz DR. Outcomes of laryngeal reinnervation for unilateral vocal fold paralysis in children: associations with age and time since injury. Ann Otol Rhinol Laryngol. 2016;125(5):433-8.

This article looks at the impact of age and time since injury on the outcomes of recurrent laryngeal nerve reinnervation. The authors feel this provides the valuable information that there was no correlation between age at surgery. There was a slight negative correlation shown with time since injury, but voice improvement was seen in all patients.

53. Hirano M, Nosoe I, et al. Electromyography for laryngeal paralysis. Neurolaryngology: recent advances. 1st ed. Boston: College-Hill; 1987. p. 232-48.

54. Thumfard W. Electromyography of the larynx. In: Samii M, Gannetta PJ, editors. The cranial nerves. 1st ed. 1981. p. 597-606. 